\title{
Metastasis to gallbladder and adrenal gland of renal cell carcinoma
}

\author{
SUNAO SHOJI ${ }^{1}$, MASAYA MUKAI ${ }^{2}$, NAOKI YAZAWA ${ }^{2}$, YASUTOMO SEKIDO $^{3}$, \\ YOSHIHIRO NAGATA ${ }^{1}$, TOYOAKI UCHIDA ${ }^{1}$ and TOSHIRO TERACHI ${ }^{4}$ \\ Departments of ${ }^{1}$ Urology, ${ }^{2}$ Surgery, ${ }^{3}$ Pathology, Tokai University Hachioji Hospital, Tokyo; \\ ${ }^{4}$ Department of Urology, Tokai University School of Medicine, Kanagawa, Japan
}

Received January 08, 2010; Accepted March 3, 2010

DOI: 10.3892/ol_00000089

\begin{abstract}
We studied a case of metastasis to the gallbladder and left adrenal gland of clear cell-type renal cell carcinoma (RCC) in the right kidney. A polypoid gallbladder tumor and left adrenal mass were found 2 years after surgery for clear cell-type RCC in a 50-year-old man. The gallbladder tumor and left adrenal mass showed hypervascularity on diagnostic imaging. Systemic image screening showed no other metastatic lesion. Simple cholecystectomy and left adrenalectomy were performed. A histopathological examination showed tumor cells in a gallbladder polyp. Furthermore, based on various specific and immunohistochemical studies, the patient was pathologically diagnosed to have gallbladder and right adrenal gland metastasis of clear cell-type RCC.
\end{abstract}

\section{Introduction}

Renal cell carcinoma (RCC) is known to metastasize to distant organs such as the lung, lymph nodes, bone, liver and adrenal glands (1). However, gallbladder involvement in patients with RCC is extremely rare. The gallbladder was identified as a metastatic site in only 4 out of 687 cases $(0.58 \%)$ in large autopsy reviews (2). Patients with distant metastasis from RCC have a poor prognosis, with a 5-year survival rate of $<10 \%$ (3). However, curative resection of metastasis in selected patients may improve long-term survival (4) in patients with gallbladder and one-sided adrenal metastasis of RCC. We presented a case of metachronous metastasis to the gallbladder and left adrenal gland from clear cell-type RCC.

Correspondence to: Dr Sunao Shoji, Department of Urology, Tokai University Hachioji Hospital, Ishikawa-machi 1838, Hachioji, Tokyo 192-0032, Japan

E-mail: sunashoj@mail.goo.ne.jp

Abbreviations: RCC, renal cell carcinoma

Key words: renal cell carcinoma, adrenal metastasis, gallbladder metastasis

\section{Case report}

A 50-year-old man was diagnosed with a gallbladder tumor during a postoperative follow-up by computed tomography (CT) in January 2009. The patient had a history of radical nephrectomy for right RCC in 2006. The pathological finding of renal tumor was clear cell-type RCC, pT1aNOM0 according to the AJCC classification of cancer staging (5). An enhanced abdominal CT showed three enlarged polypoid masses with a mosaic pattern and maximum tumor size of $11 \times 8 \mathrm{~mm}$ in diameter in-filling the gallbladder. The CT also showed an enhanced mass with a mosaic pattern of $13 \times 19 \mathrm{~mm}$ in diameter in the left adrenal gland. Magnetic resonance imaging (MRI) revealed a polypoid lesion in the gallbladder and a mass in the left adrenal gland. The polypoid lesion in the gallbladder showed a low signal intensity on a T1-weighted MRI image and an iso signal intensity on a T2-weighted MRI image, but no suspicious findings with tumor invasion to the gallbladder muscle layer (Fig. 1). Similar to the polypoid lesion in the gallbladder, the mass in the left adrenal gland showed a low signal intensity on a T1-weighted MRI image and an iso signal intensity on a T2-weighted MRI image. Blood biochemistry results were in the normal ranges, including carcinoembryonic antigen (CEA) and carbohydrate antigen 19-9 (CA19-9). The result of the systemic image screening was that another metastatic lesion was not found.

Under the diagnosis of metastatic left adrenal tumor and presumptive diagnosis of metastatic gallbladder tumor from RCC, the patient underwent simple cholecystectomy and left adrenalectomy. Macroscopically, the resected specimen of the gallbladder tumor showed an 11x9 mm (Fig. 2), 7x9 mm and $8 \times 8 \mathrm{~mm}$-sized polypoid lesion, and the adrenal mass showed a 12x16x13-diameter tumor. Microscopically, the gallbladder tumor was located in the submucosal layer, lifting the surrounding mucosa of the gallbladder (Fig. 3). The gallbladder mucosal cells had no atypia.

The tumor cells had round uniform nuclei, clear cytoplasm and well-defined cytoplasmic borders, forming alveolar patterns (Fig. 4). Neither foci of mucin production nor ordinary adenocarcinoma patterns of digestive organs were noted. Immunohistochemically, the tumor cells were negative for cytokeratin 7 (CK7) and CEA, but positive for vimentin. Adrenal tumor cell findings were similar to the renal tumor 


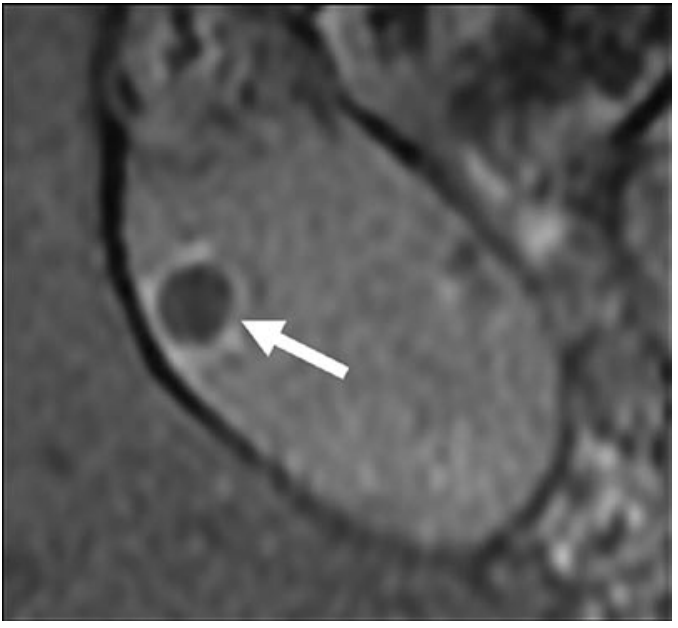

Figure 1. The polypoid lesion in the gallbladder showed a low signal intensity on a T1-weighted MRI image. No suspicious findings of tumor invasion to the muscle layer of the gallbladder were noted.

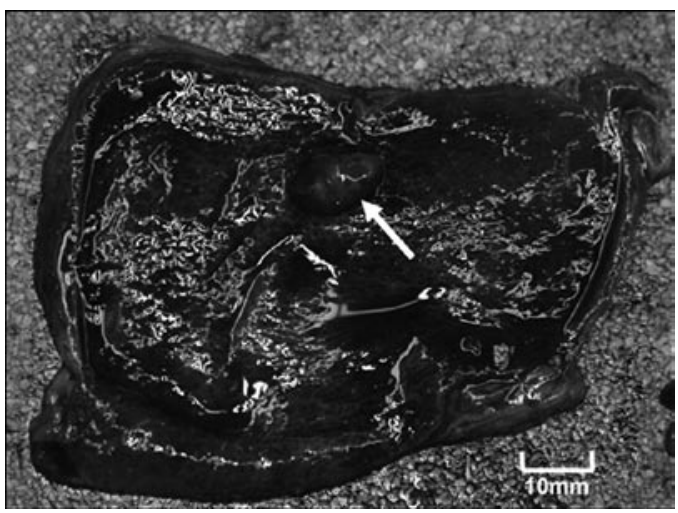

Figure 2. The resected specimen of the maximum gallbladder tumor showed an $11 \times 9 \mathrm{~mm}$-sized polypoid lesion.

microscopically. Therefore, the final diagnosis was metastasis to the gallbladder and adrenal gland from clear cell-type RCC. Pathological findings of the gallbladder tumor and adrenal tumors showed clear cell carcinoma. The surgical specimen of the gallbladder and adrenal tumor showed histological evidence of clear cell carcinoma with the same characteristics as those of the primary renal tumor. The patient showed no sign of recurrence 8 months after the last surgery and was without any additional therapies.

\section{Discussion}

RCC is known to metastasize mainly to the lungs, lymph nodes, bone, brain, liver, adrenal glands and the other kidney, and rarely to organs such as the vertebrae, stomach, spleen, pancreas and diaphragm (1). However, gallbladder metastasis of RCC is seldom detected, being present in $0.58 \%$ of autopsies (2). In a review of the literature, we found 15 reported cases of gallbladder metastasis of RCC (6-8).

Dynamic contrast-enhanced CT is useful in the differential diagnosis of a metastatic gallbladder tumor from RCC and a primary gallbladder carcinoma because the former is

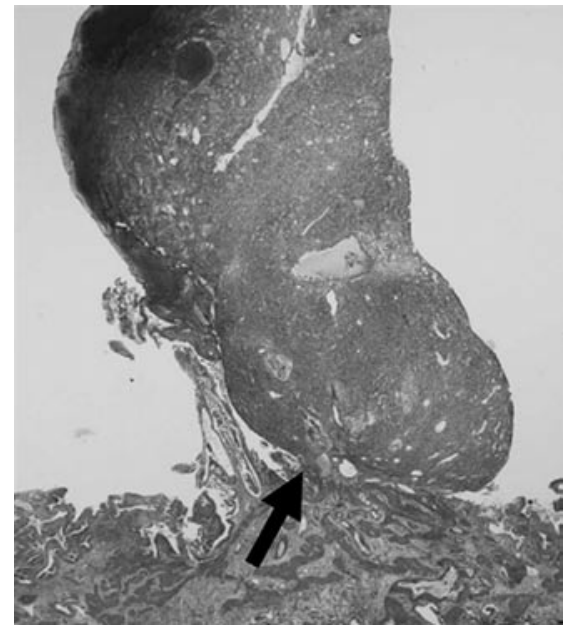

Figure 3. Microscopically, the gallbladder tumor was located in the submucosal layer, lifting the surrounding mucosa of the gallbladder (H\&E stain; magnification, $\mathrm{x} 12.5)$.

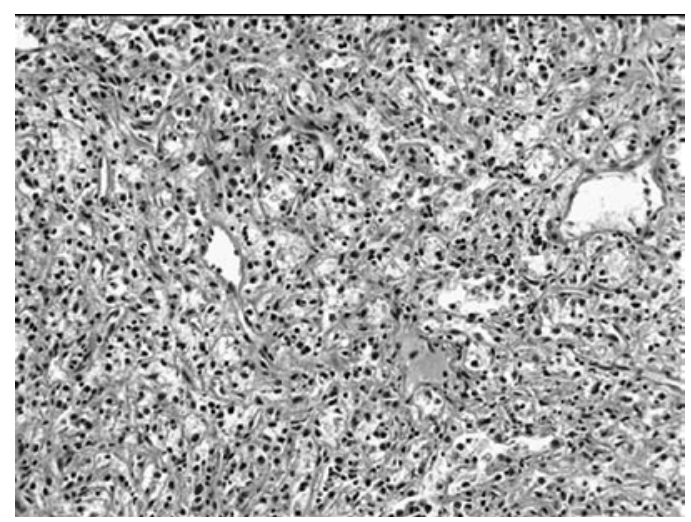

Figure 4. Microscopically, the gallbladder mucosal cells had no atypia. The tumor cells had round uniform nuclei, clear cytoplasm, and well-defined cytoplasmic borders, forming alveolar patterns (H\&E stain; magnification, $\mathrm{x} 400$ ).

hypervascular. In the present patient, contrast-enhanced CT showed a high density on an arterial dominant phase image, and the MRI showed a high signal intensity on a T2-weighted image. In the literature, from macroscopic findings, 9 out of 15 cases $(60 \%)$ were polypoid type and 3 out of 15 cases (20\%) were pedunculated type. To determine the final differential diagnosis between primary and secondary gallbladder tumors, an immunohistochemical evaluation was required. Immunohistochemically, the primary clear cell carcinoma of the gallbladder is strongly positive for CEA and CK7 and moderately positive for CK10, but negative for vimentin. On the other hand, metastatic RCC of the gallbladder is positive for vimentin, but negative for CEA, CK7 and CK10 (9). Based on the immunohistochemical findings, our final diagnosis was metastatic gallbladder tumor from RCC as opposed to primary clear cell carcinoma of the gallbladder.

Follow-up information on this series was insufficient to demonstrate the efficacy of cholecystectomy for the metastasis of RCC. However, cholecystectomy for curative resection of metastatic RCC may be advocated since, following curative resection of second and third metastasis, the survival rates have not been found to be different from those after 
first metastatectomy (4). Notably, no patients who underwent simple cholecystectomy were reported to develop local recurrence in the liver or the bile duct. Hematogenous metastasis to the gallbladder occurs as small flat nodules below the mucosal layer which grow as polyps. Therefore, we consider that simple cholecystectomy may be sufficient for curative resection of gallbladder metastasis from RCC if there is no invasion of the muscle layer of the gallbladder. In our case, a simple cholecystectomy was performed since no suspicious findings of tumor invasion to the muscle layer of the gallbladder in pre-operative imaging and intra-operative findings were noted.

In conclusion, RCC can metastasize to the gallbladder, and this should be considered in patients with hypervascular polypoid or pedunculated-type gallbladder tumors who have a previous history of RCC. Pre-operative imaging and intra-operative findings are important factors in deciding on adequate treatment.

\section{References}

1. Saitoh H: Distant metastasis of renal adenocarcinoma. Cancer 48: 1487-1491, 1981.

2. Weiss L, Harlos J, Torhorst J, et al: Metastatic patterns of renal cell carcinoma: an analysis of 687 necropsies. Cancer Res Clin Oncol 114: 605-612, 1988.
3. Vogelzang $\mathrm{N}$ and Stadler W: Kidney cancer. Lancet 352: 1691-1696, 1998.

4. Kavolius J, Mastorakos D, Pavlovich C, et al: Resection of metastasis renal cell carcinoma. J Clin Oncol 16: 2261-2266, 1998.

5. Greene F, Page D, Fleming I, et al: AJCC Cancer Staging Manual. 6th edition. Springer-Verlag, New York, 2002.

6. Nojima H, Cho A, Yamamoto H, et al: Renal cell carcinoma with unusual metastasis to the gallbladder. J Hepatobiliary Pancreat Surg 15: 209-212, 2008.

7. Ricci V, Carbone S, Testi W, et al: Single gallbladder and multiple pancreatic metastases from renal cell carcinoma sixteen years after nephrectomy. Chir Ital 60: 311-314, 2008.

8. Sand M, Bechara FG, Kopp J, et al: Gallbladder metastasis from renal cell carcinoma mimicking acute cholecystitis. Eur J Med Res 14: 90-92, 2009.

9. Bittinger A, Alterkrugar I and Barth P: Clear cell carcinoma of the gallbladder. A histological and immunohistochemical study. Pathol Res Pract 191: 1259-1265, 1995. 